Pacific

Journal of

Mathematics

A NOTE ON GENERALIZED BERNOULLI NUMBERS

Kwang-Wu Chen and Minking Eie 


\title{
A NOTE ON GENERALIZED BERNOULLI NUMBERS
}

\author{
Kwang-Wu Chen and Minking Eie
}

In this paper, we consider the zeta function $Z(P, \chi, s)$ associated with a polynomial $P(X) \in \mathbb{R}\left[X_{1}, \ldots, X_{r}\right]$ and $\chi=$ $\left(\chi_{1}, \ldots, \chi_{r}\right)$ with $\chi_{j}$ non-trivial Dirichlet characters, defined by

$$
Z(P, \chi, s)=\sum_{n_{1}=1}^{\infty} \cdots \sum_{n_{r}=1}^{\infty} \chi_{1}\left(n_{1}\right) \cdots \chi_{r}\left(n_{r}\right) P\left(n_{1}, \ldots, n_{r}\right)^{-s}
$$

which is absolutely convergent for sufficiently large Re $s$ under some conditions on $P(X)$. We shall prove that the special value $Z(P, \chi,-m)$ is completely determined by $P^{m}(X)$ in a simple way. As an immediate application, we give a closed expression for sums of products of any number of generalized Bernoulli numbers.

\section{Introduction and Notation.}

As usual, $\mathbb{N}$ denotes the set of positive numbers, $\mathbb{N}_{0}=\mathbb{N} \bigcup\{0\}$ and $\mathbb{R}$ denotes the field of real numbers. Let $\chi$ be a non-trivial Dirichlet character with conductor $N$. The $L$-series attached to $\chi$ is defined by

$$
L(s, \chi)=\sum_{n=1}^{\infty} \chi(n) n^{-s}, \quad \operatorname{Re} s>1 .
$$

It is well known [14] that $L(s, \chi)$ may be continued analytically to the whole complex $s$-plane. Furthermore, the special values at non-positive integers $s=-m(m=0,1,2, \ldots)$ can be expressed by the generalized Bernoulli numbers $B_{\chi}^{n}(n=0,1,2, \ldots)$ defined by

$$
\sum_{a=1}^{N} \frac{\chi(a) t e^{a t}}{e^{N t}-1}=\sum_{n=0}^{\infty} \frac{B_{\chi}^{n} t^{n}}{n !},|t|<\frac{2 \pi}{N} .
$$

Indeed, $L(-m, \chi)=-\frac{B_{\chi}^{m+1}}{m+1}$ as given on Page 30 of [14]. The generalized Bernoulli numbers can be expressed in terms of Bernoulli polynomials as

$$
B_{\chi}^{n}=N^{n-1} \sum_{a=1}^{N} \chi(a) B_{n}\left(\frac{a}{N}\right)
$$


where the Bernoulli polynomials $B_{n}(X)$ are defined by

$$
\frac{t e^{X t}}{e^{t}-1}=\sum_{n=0}^{\infty} B_{n}(X) \frac{t^{n}}{n !},|t|<2 \pi \text {. }
$$

Also

$$
B_{n}(X)=\sum_{k=0}^{n}\left(\begin{array}{l}
n \\
k
\end{array}\right) B_{n-k} X^{k}
$$

where the Bernoulli numbers $B_{n}(n=0,1,2, \ldots)$ are defined by

$$
\frac{t}{e^{t}-1}=\sum_{n=0}^{\infty} B_{n} \frac{t^{n}}{n !}, \quad|t|<2 \pi \text {. }
$$

Consequently, we can express the generalized Bernoulli numbers in terms of Bernoulli numbers as follows:

$$
B_{\chi}^{n}=\sum_{a=1}^{N} \chi(a) \sum_{k=0}^{n}\left(\begin{array}{l}
n \\
k
\end{array}\right) B_{k} a^{n-k} N^{k-1} .
$$

Let $P(X)=P\left(X_{1}, \ldots, X_{r}\right)$ be a polynomial of $r$ variables with non-negative real coefficients such that $P(n)>0$ for all $n \in \mathbb{N}^{r}$ and the series

$$
\sum_{n \in \mathbb{N}^{r}} P(n)^{-s}=\sum_{n_{1}=1}^{\infty} \cdots \sum_{n_{r}=1}^{\infty} P\left(n_{1}, \ldots, n_{r}\right)^{-s}
$$

is absolutely convergent for $\operatorname{Re} s>\sigma>0 . \chi_{1}, \ldots, \chi_{r}$ are non-trivial Dirichlet characters with conductors $N_{1}, \ldots, N_{r}$, respectively. Consider the zeta function associated with $P$ and $\chi=\left(\chi_{1}, \ldots, \chi_{r}\right)$ defined by

$$
Z(P, \chi, s)=\sum_{n_{1}=1}^{\infty} \cdots \sum_{n_{r}=1}^{\infty} \chi_{1}\left(n_{1}\right) \cdots \chi_{r}\left(n_{r}\right) P\left(n_{1}, \ldots, n_{r}\right)^{-s}, \text { Re } s>\sigma .
$$

It is the main purpose of this paper to prove the following result.

Theorem. $Z(P, \chi, s)$ defined above has a meromorphic analytic continuation to the whole complex s-plane. For any integer $m \geq 0$, if

$$
P^{m}(X)=\sum_{|\alpha|=0}^{m p} C_{\alpha} X_{1}^{\alpha_{1}} \cdots X_{r}^{\alpha_{r}}, \quad p=\operatorname{deg} P
$$

then

$$
\begin{aligned}
Z(P, \chi,-m) & =\sum_{|\alpha|=0}^{m p} C_{\alpha} L\left(-\alpha_{1}, \chi_{1}\right) \cdots L\left(-\alpha_{r}, \chi_{r}\right) \\
& =(-1)^{r} \sum_{|\alpha|=0}^{m p} C_{\alpha} \prod_{j=1}^{r} \frac{B_{\chi_{j}}^{\alpha_{j}+1}}{\alpha_{j}+1} .
\end{aligned}
$$


Another zeta function $Z(P, \xi, s)$ defined by

$$
Z(P, \xi, s)=\sum_{n_{1}=1}^{\infty} \cdots \sum_{n_{r}=1}^{\infty} \xi_{1}^{n_{1}} \cdots \xi_{r}^{n_{r}} P\left(n_{1}, \ldots, n_{r}\right)^{-s}
$$

was considered by P. Cassou-Nouguès in [2]. Her result for the special values of $Z(P, \xi, s)$ can be restated as

$$
Z(P, \xi,-m)=\lim _{t \rightarrow 0^{+}} \sum_{n_{1}=1}^{\infty} \cdots \sum_{n_{r}=1}^{\infty} \xi_{1}^{n_{1}} \cdots \xi_{r}^{n_{r}} P^{m}(n) e^{-\left(n_{1}+\cdots+n_{r}\right) t} .
$$

Here we also have the same formula for the special values of $Z(P, \chi, s)$, i.e.,

$$
Z(P, \chi,-m)=\lim _{t \rightarrow 0^{+}} \sum_{n_{1}=1}^{\infty} \cdots \sum_{n_{r}=1}^{\infty} \chi_{1}\left(n_{1}\right) \cdots \chi_{r}\left(n_{r}\right) P^{m}(n) e^{-\left(n_{1}+\cdots+n_{r}\right) t} .
$$

However $\chi(n)=\prod_{j=1}^{r} \chi_{j}\left(n_{j}\right)$ is a multiplicative character while $\xi^{n}$ $=\prod_{j=1}^{r} \xi_{j}^{n_{j}}$ is an additive character. Hence the treatments are different in some respect. As shown in Section 4, P. Cassou-Nouguès' formula for the special values of $Z(P, \xi, s)$ follows from our formula for the special values of $Z(P, \chi, s)$. In additon we have another explicit expression for the special values of $Z(P, \xi, s)$.

A well-known relation among the Bernoulli numbers is

$$
\sum_{k=1}^{n-1}\left(\begin{array}{l}
2 n \\
2 k
\end{array}\right) B_{2 k} B_{2 n-2 k}=-(2 n+1) B_{2 n}, \quad \text { for } n \geq 2 .
$$

This was found by many authors, including Euler (ref. [5], [8]). Dilcher [5] generalized the formula for sums of products of any number of both Bernoulli and Euler numbers. Bernoulli and Euler numbers are special cases of the generalized Bernoulli numbers $B_{\chi}^{n}$ belonging to a residue class character $\chi$. However it is not easy to get the generalized formula for generalized Bernoulli numbers. At the end of this paper, we give a closed expression for the case as an immediate application of our main theorem.

\section{Some Basic Results.}

We need some classical results reproduced in [15].

Proposition 1. Suppose that $\varphi(s)=\sum_{\lambda>0} a_{\lambda} \lambda^{-s}$ ( $\lambda$ ranges over a sequence of positive real numbers tending $+\infty)$ is a Dirichlet series converging for sufficiently large Re $s . f(t)=\sum_{\lambda>0} a_{\lambda} e^{-\lambda t}$ is the corresponding exponential series. Suppose that at $t=0, f(t)$ has the asymptotic expansion

$$
\sum_{n \geq 0} C_{n} t^{n / p}
$$

where $p$ is a fixed positive number. Then: 
(1) $\varphi(s)$ has a meromorphic continuation to the whole complex plane.

(2) $\varphi(s)$ has possible simple poles at $s=-n / p$, where $n$ is not a multiple of $p$, with residue $C_{n} / \Gamma(-n / p)$, and has no other poles.

(3) $\varphi(-n)=(-1)^{n} n ! C_{n p}$.

Note that the above proposition is different from Proposition 2 of [15]. However, it follows from

$$
\begin{aligned}
\varphi(s) \Gamma(s) & =\int_{0}^{\infty} t^{s-1} f(t) d t, \quad \operatorname{Re} s>\sigma \\
& =\int_{0}^{\delta} t^{s-1} \sum_{n=0}^{\infty} C_{n} t^{n / p} d t+\int_{\delta}^{\infty} t^{s-1} f(t) d t \\
& =\sum_{n=0}^{\infty} C_{n} \frac{\delta^{s+\frac{n}{p}}}{s+\frac{n}{p}}+\int_{\delta}^{\infty} t^{s-1} f(t) d t
\end{aligned}
$$

where $\delta$ is a small positive number so that $f(t)=\sum_{n=0}^{\infty} C_{n} t^{n / p}$. From the above, we get our assertions.

A function $f(x)$ is called a rapidly decreasing function if it belongs to $C^{\infty}\left(\mathbb{R}^{n}\right)$ and satisfies

$$
\lim _{|x| \rightarrow \infty}|x|^{k}\left|D^{\alpha} f(x)\right|=0
$$

for any $\alpha$ and any integer $k>0$ (ref. [10], or page 245 in [11]). The following is a consequence of the Euler-Maclaurin summation formula which is also reproduced in [15].

Proposition 2. Suppose that $f$ is a rapidly decreasing function on $[0, \infty)$ and at $t=0, f$ has the power series expansion

$$
f(t)=\sum_{r=0}^{\infty} \frac{f^{(r)}(0)}{r !} t^{r}
$$

Suppose that $g(t)=\sum_{n=1}^{\infty} f(n t)$. Then at $t=0, g(t)$ has the asymptotic expansion

$$
\frac{C}{t}+\sum_{r=0}^{\infty}(-1)^{r} \frac{B_{r+1}}{(r+1) !} f^{(r)}(0) t^{r} \quad \text { with } \quad C=\int_{0}^{\infty} f(t) d t .
$$

To find the special value at $s=-m$ of the zeta function

$$
Z(P, \chi, s)=\sum_{n_{1}=1}^{\infty} \cdots \sum_{n_{r}=1}^{\infty} \chi_{1}\left(n_{1}\right) \cdots \chi_{r}\left(n_{r}\right) P(n)^{-s},
$$

by Proposition 1, it is equivalent to find the coefficient of $t^{m}$ in the asymptotic expansion at $t=0$ of the function

$$
\sum_{n_{1}=1}^{\infty} \cdots \sum_{n_{r}=1}^{\infty} \chi_{1}\left(n_{1}\right) \cdots \chi_{r}\left(n_{r}\right) \exp \{-P(n) t\}
$$


It is also equivalent to find the constant term in the asymptotic expansion at $t=0$ of the function

$$
g(t)=\sum_{n_{1}=1}^{\infty} \cdots \sum_{n_{r}=1}^{\infty} \chi_{1}\left(n_{1}\right) \cdots \chi_{r}\left(n_{r}\right) P^{m}(n) \exp \{-P(n) t\} .
$$

For the given polynomial

$$
P(X)=\sum_{|\alpha|=0}^{p} A_{\alpha} X^{\alpha}, \quad p=\operatorname{deg} P,
$$

we let

$$
Q(X, Y)=\sum_{|\alpha|=0}^{p} A_{\alpha} X^{\alpha} Y^{p-|\alpha|}
$$

be the corresponding homogeneous polynomial in $r+1$ variables. Obviously, $Q(n t, t)=P(n) t^{p}$ and so

$$
\begin{aligned}
g\left(t^{p}\right) & =\sum_{n \in \mathbb{N}^{r}} \chi_{1}\left(n_{1}\right) \cdots \chi_{r}\left(n_{r}\right) P^{m}(n) \exp \left\{-P(n) t^{p}\right\} \\
& =\sum_{n \in \mathbb{N}^{r}} \chi_{1}\left(n_{1}\right) \cdots \chi_{r}\left(n_{r}\right) P^{m}(n) \exp \{-Q(n t, t)\} \\
& =\sum_{|\alpha|=0}^{m p} C_{\alpha} \sum_{n \in \mathbb{N}^{r}} \chi_{1}\left(n_{1}\right) \cdots \chi_{r}\left(n_{r}\right) n^{\alpha} \exp \{-Q(n t, t)\}
\end{aligned}
$$

where

$$
P^{m}(X)=\sum_{|\alpha|=0}^{m p} C_{\alpha} X^{\alpha} \quad \text { and } \quad n^{\alpha}=n_{1}^{\alpha_{1}} \cdots n_{r}^{\alpha_{r}} .
$$

In the next section, we shall compute the asymptotic expansion at $t=0$ of the function

$$
f_{\beta}(t)=\sum_{n \in \mathbb{N}^{r}} \chi_{1}\left(n_{1}\right) \cdots \chi_{r}\left(n_{r}\right) n^{\beta} \exp \{-Q(n t, t)\} .
$$

\section{The Proof of the Theorem.}

First we shall prove the case $r=1$. Indeed this special case plays an important role in our proof of the theorem.

Lemma 1. Let $P$ be a polynomial with real coefficients such that $P(n)>0$ for all $n \in \mathbb{N}$ and $Q$ be the corresponding polynomial defined above. Let 
$h(x, t)=x^{\beta} \exp \{-Q(x t, t)\}, N$ a positive integer and $1 \leq j \leq N$. Furthermore, denote

$$
\begin{aligned}
f_{j}(t) & =\sum_{k=0}^{\infty} h(k N+j, t) \\
& =\sum_{k=0}^{\infty}(k N+j)^{\beta} \exp \{-Q((k N+j) t, t)\} .
\end{aligned}
$$

Then

$$
f_{j}(t)=h(j, t)+\frac{1}{N} \int_{j}^{\infty} h(x, t) d x+\sum_{r=0}^{\infty} \frac{(-1)^{r} B_{r+1}}{(r+1) !} h^{(r)}(j, t) N^{r}
$$

where $h^{(r)}(x, t)$ is the $r$-th partial derivative with respect to $x$.

Proof. It follows from the Euler-Maclaurin summation formula that

$$
\begin{aligned}
\sum_{k=1}^{\infty} h(k N+j, t)= & \int_{0}^{\infty}(N x+j)^{\beta} \exp \{-Q((N x+j) t, t)\} d x \\
& +\sum_{r=0}^{\infty} \frac{(-1)^{r} B_{r+1}}{(r+1) !} h^{(r)}(j, t) N^{r} \\
= & \frac{1}{N} \int_{j}^{\infty} h(x, t) d x+\sum_{r=0}^{\infty} \frac{(-1)^{r} B_{r+1}}{(r+1) !} h^{(r)}(j, t) N^{r} .
\end{aligned}
$$

Proposition 3. Let $\chi$ be a non-trivial character with conductor $N$. Let $\beta \geq 0$ be an integer, and $P, Q$ polynomials as given in the previous lemma. Suppose that

$$
f(t)=\sum_{n=1}^{\infty} \chi(n) n^{\beta} \exp \{-Q(n t, t)\}
$$

Then

$$
\begin{aligned}
f(t)=\sum_{j=1}^{N} \chi(j) h(j, t)-\frac{1}{N} \sum_{j=1}^{N} \chi(j) & \int_{0}^{j} h(x, t) d x \\
& +\sum_{j=1}^{N} \chi(j) \sum_{r=0}^{\infty} \frac{(-1)^{r} B_{r+1}}{(r+1) !} h^{(r)}(j, t) N^{r} .
\end{aligned}
$$

In particular at $t=0, f(t)$ has an asymptotic expansion of the form

$$
\sum_{n=0}^{\infty} d_{n} t^{n}
$$


with the constant term $d_{0}$ given by

$$
d_{0}=-\frac{B_{\chi}^{\beta+1}}{\beta+1}=L(-\beta, \chi) .
$$

Proof. Note that

$$
\begin{aligned}
f(t) & =\sum_{j=1}^{N} \chi(j) \sum_{k=0}^{\infty}(N k+j)^{\beta} \exp \{-Q((N k+j) t, t)\} \\
& =\sum_{j=1}^{N} \chi(j) f_{j}(t) .
\end{aligned}
$$

So the first assertion follows from Lemma 1 by noting that

$$
\int_{j}^{\infty} h(x, t) d x=\int_{0}^{\infty} h(x, t) d x-\int_{0}^{j} h(x, t) d x
$$

and

$$
\sum_{j=1}^{N} \chi(j) \int_{j}^{\infty} h(x, t) d x=-\sum_{j=1}^{N} \chi(j) \int_{0}^{j} h(x, t) d x
$$

since $\sum_{j=1}^{N} \chi(j)=0$. Also, from this expression of $f(t)$ we have a power series expansion of the form

$$
\sum_{n=0}^{\infty} d_{n} t^{n}
$$

with

$$
\begin{aligned}
d_{0}= & \sum_{j=1}^{N} \chi(j) h(j, 0)-\frac{1}{N} \sum_{j=1}^{N} \chi(j) \int_{0}^{j} h(x, 0) d x \\
& +\sum_{j=1}^{N} \chi(j) \sum_{r=0}^{\infty} \frac{(-1)^{r} B_{r+1}}{(r+1) !} h^{(r)}(j, 0) N^{r} \\
= & \sum_{j=1}^{N} \chi(j) j^{\beta}-\frac{1}{N} \sum_{j=1}^{N} \chi(j) \frac{j^{\beta+1}}{\beta+1}+\sum_{j=1}^{N} \chi(j) \sum_{r=0}^{\infty} \frac{(-1)^{r} B_{r+1}}{(r+1) !} h^{(r)}(j, 0) N^{r} .
\end{aligned}
$$

Now it remains to compute $h^{(r)}(j, 0)$. The Leibniz rule for differentiation yields that

$$
\begin{aligned}
D_{x}^{r} h(x, t) & =D_{x}^{r}\left[x^{\beta} \exp \{-Q(x t, t)\}\right] \\
& =\sum_{u=0}^{r}\left(\begin{array}{l}
r \\
u
\end{array}\right) D_{x}^{u}\left(x^{\beta}\right) D_{x}^{r-u} \exp \{-Q(x t, t)\} .
\end{aligned}
$$


From the above, we see that

$$
\left.D_{x}^{r} h(x, t)\right|_{x=j, t=0}= \begin{cases}\frac{\beta !}{(\beta-r) !} j^{\beta-r}, & \text { if } r \leq \beta \\ 0, & \text { otherwise. }\end{cases}
$$

It follows that

$$
\begin{aligned}
d_{0}=\sum_{j=1}^{N} \chi(j) j^{\beta}-\frac{1}{N} \sum_{j=1}^{N} \chi(j) & \frac{j^{\beta+1}}{\beta+1} \\
& +\sum_{j=1}^{N} \chi(j) \sum_{r=0}^{\beta} \frac{(-1)^{r} \beta !}{(r+1) !(\beta-r) !} B_{r+1} N^{r} j^{\beta-r} .
\end{aligned}
$$

Note that $B_{1}=-\frac{1}{2}$ and $(-1)^{r} B_{r+1}=-B_{r+1}$ if $r \geq 1$. So

$$
\begin{aligned}
d_{0} & =-\frac{1}{N} \sum_{j=1}^{N} \chi(j) \frac{j^{\beta+1}}{\beta+1}-\sum_{j=1}^{N} \chi(j) \sum_{r=0}^{\beta} \frac{\beta !}{(r+1) !(\beta-r) !} B_{r+1} N^{r} j^{\beta-r} \\
& =-\frac{B_{\chi}^{\beta+1}}{\beta+1} .
\end{aligned}
$$

Our theorem is a direct consequence of the following proposition.

Proposition 4. Let $\chi=\left(\chi_{1}, \ldots, \chi_{r}\right), \beta=\left(\beta_{1}, \ldots, \beta_{r}\right), P$ and $Q$ as given in Section 2. Suppose that

$$
f_{\beta}(t)=\sum_{n_{1}=1}^{\infty} \cdots \sum_{n_{r}=1}^{\infty} \chi_{1}\left(n_{1}\right) \cdots \chi_{r}\left(n_{r}\right) n^{\beta} \exp \{-Q(n t, t)\} .
$$

Then $f_{\beta}(t)$ has an asymptotic expansion of the form

$$
\sum_{n=0}^{\infty} d_{n} t^{n}
$$

with the constant term $d_{0}$ given by

$$
\begin{aligned}
d_{0} & =L\left(-\beta_{1}, \chi_{1}\right) \cdots L\left(-\beta_{r}, \chi_{r}\right) \\
& =(-1)^{r} \prod_{j=1}^{r} \frac{B_{\chi_{j}}^{\beta_{j}+1}}{\beta_{j}+1} .
\end{aligned}
$$

Proof. We prove the assertion by induction on $r$. The case $r=1$ was already proved in the previous proposition. Suppose that $r \geq 2$ and the assertion is true for the case of $r-1$ variables. Consider the case of $r$ variables. Applying 
the previous proposition to the first summation of $f_{\beta}(t)$, where $n_{1}$ ranges over all positive integers, we obtain

$$
\begin{aligned}
f_{\beta}(t)=\sum_{j=1}^{N_{1}} \chi_{1}(j) j^{\beta_{1}} h(j, t)-\frac{1}{N_{1}} \sum_{j=1}^{N_{1}} & \chi_{1}(j) \int_{0}^{j} x^{\beta_{1}} h(x, t) d x \\
& +\sum_{j=1}^{N_{1}} \chi_{1}(j) \sum_{r=0}^{\infty} \frac{(-1)^{r} B_{r+1}}{(r+1) !} \tilde{h}_{j}^{(r)}(0, t)
\end{aligned}
$$

where

$$
\begin{aligned}
h(x, t)=\sum_{n_{2}=1}^{\infty} \cdots \sum_{n_{r}=1}^{\infty} \chi_{2}\left(n_{2}\right) \cdots \chi_{r}\left(n_{r}\right) n_{2}^{\beta_{2}} \cdots n_{r}^{\beta_{r}} \\
\cdot \exp \left\{-Q\left(x t, n_{2} t, \ldots, n_{r} t, t\right)\right\},
\end{aligned}
$$

and

$$
\begin{aligned}
\tilde{h}_{j}(x, t) & =\left(N_{1} x+j\right)^{\beta_{1}} \sum_{n_{2}=1}^{\infty} \cdots \sum_{n_{r}=1}^{\infty} \chi_{2}\left(n_{2}\right) \cdots \chi_{r}\left(n_{r}\right) n_{2}^{\beta_{2}} \cdots n_{r}^{\beta_{r}} \\
\cdot \exp \left\{-Q\left(\left(N_{1} x+j\right) t, n_{2} t, \ldots, n_{r} t, t\right)\right\} & \\
= & \left(N_{1} x+j\right)^{\beta_{1}} h\left(N_{1} x+j, t\right) .
\end{aligned}
$$

Note that

$$
Q\left(\alpha t, n_{2} t, \ldots, n_{r} t, t\right)=P\left(\alpha, n_{2}, \ldots, n_{r}\right) t^{p^{\prime}}, \quad p^{\prime}=\operatorname{deg} P\left(\alpha, X_{2}, \ldots, X_{r}\right)
$$

for any fixed number $\alpha>0$. Applying our induction hypothesis to $h(j, t)$, $h(x, t)$, and $\tilde{h}_{j}(x, t)$, we get the asymptotic expansion of $f_{\beta}(t)$, and the constant term $d_{0}$ is

$$
\begin{aligned}
d_{0}= & f_{\beta}(0) \\
= & \sum_{j=1}^{N_{1}} \chi_{1}(j) j^{\beta_{1}} h(j, 0)-\frac{1}{N_{1}} \sum_{j=1}^{N_{1}} \chi_{1}(j) \int_{0}^{j} x^{\beta_{1}} h(x, 0) d x \\
& +\sum_{j=1}^{N_{1}} \chi_{1}(j) \sum_{r=0}^{\infty} \frac{(-1)^{r} B_{r+1}}{(r+1) !} \tilde{h}_{j}^{(r)}(0,0) .
\end{aligned}
$$

To compute $\tilde{h}_{j}^{(r)}(0,0)$, we use a trick similar to the one in Proposition 3 for computing $h^{(r)}(j, 0)$. The Leibniz rule for differentiation yields that

$$
D_{x}^{r} \tilde{h}_{j}(x, t)=\sum_{u=0}^{r}\left(\begin{array}{l}
r \\
u
\end{array}\right) D_{x}^{u}\left[\left(N_{1} x+j\right)^{\beta_{1}}\right] D_{x}^{r-u}\left[h\left(N_{1} x+j, t\right)\right] .
$$


From the above, we see that

$$
\left.D_{x}^{r} \tilde{h}_{j}(x, t)\right|_{x=0, t=0}= \begin{cases}\frac{\beta_{1} ! N_{1}^{r}}{\left(\beta_{1}-r\right) !} j^{\beta_{1}-r} h(j, 0), & \text { if } r \leq \beta_{1} \\ 0, & \text { otherwise. }\end{cases}
$$

It follows that

$$
\begin{aligned}
d_{0}=\sum_{j=1}^{N_{1}} \chi_{1}(j) j^{\beta_{1}} h(j, 0) & -\frac{1}{N_{1}} \sum_{j=1}^{N_{1}} \chi_{1}(j) \int_{0}^{j} x^{\beta_{1}} h(x, 0) d x \\
& +\sum_{j=1}^{N_{1}} \chi_{1}(j) \sum_{r=0}^{\beta_{1}} \frac{(-1)^{r} B_{r+1} \beta_{1} ! N_{1}^{r} j^{\beta_{1}-r}}{(r+1) !\left(\beta_{1}-r\right) !} h(j, 0) .
\end{aligned}
$$

Since the constant term in the asymptotic expansion of $h(j, t)$ or $h(x, t)$ is

$$
(-1)^{r-1} \prod_{j=2}^{r} \frac{B_{\chi_{j}}^{\beta_{j}+1}}{\beta_{j}+1}
$$

we have

$$
\begin{aligned}
d_{0}=(-1)^{r-1} \prod_{j=2}^{r} \frac{B_{\chi_{j}}^{\beta_{j}+1}}{\beta_{j}+1}\left[\sum_{j=1}^{N_{1}} \chi_{1}(j) j^{\beta_{1}}-\frac{1}{N_{1}} \sum_{j=1}^{N_{1}} \chi_{1}(j) \frac{j^{\beta_{1}+1}}{\beta_{1}+1}\right. \\
\left.\quad+\sum_{j=1}^{N_{1}} \chi_{1}(j) \sum_{r=0}^{\beta_{1}} \frac{(-1)^{r} \beta_{1} !}{(r+1) !\left(\beta_{1}-r\right) !} B_{r+1} N^{r} j^{\beta_{1}-r}\right] \\
=(-1)^{r-1} \prod_{j=2}^{r} \frac{B_{\chi_{j}}^{\beta_{j}+1}}{\beta_{j}+1} \cdot\left(-\frac{B_{\chi_{1}}^{\beta_{1}+1}}{\beta_{1}+1}\right) \\
=(-1)^{r} \prod_{j=1}^{r} \frac{B_{\chi_{j}}^{\beta_{j}+1}}{\beta_{j}+1} .
\end{aligned}
$$

This proves our assertions.

Corollary. Suppose that

$$
F(t)=\sum_{n \in \mathbb{N}^{r}} \chi_{1}\left(n_{1}\right) \cdots \chi_{r}\left(n_{r}\right) P^{m}(n) e^{-\left(n_{1}+\cdots+n_{r}\right) t}, \quad t>0
$$

then

$$
Z(P, \chi,-m)=\lim _{t \rightarrow 0^{+}} F(t) .
$$

Proof. From the notation in our main theorem it follows that

$$
F(t)=\sum_{|\beta|=0}^{m p} C_{\beta} F_{\beta}(t)
$$


with

$$
\begin{aligned}
F_{\beta}(t) & =\sum_{n \in \mathbb{N}^{r}} \chi_{1}\left(n_{1}\right) \cdots \chi_{r}\left(n_{r}\right) n^{\beta} e^{-\left(n_{1}+\cdots+n_{r}\right) t} \\
& =\prod_{j=1}^{r}\left[\sum_{n=1}^{\infty} \chi_{j}(n) n^{\beta_{j}} e^{-n t}\right] .
\end{aligned}
$$

From

$$
\begin{aligned}
\sum_{n=1}^{\infty} \chi_{j}(n) e^{-n t} & =\sum_{k=0}^{\infty} \sum_{a=1}^{N_{j}} \chi_{j}(a) e^{-\left(a+k N_{j}\right) t} \\
& =\sum_{a=1}^{N_{j}} \frac{\chi_{j}(a) e^{-a t}}{1-e^{-N_{j} t}} \\
& =\sum_{n=1}^{\infty} \frac{-B_{\chi_{j}}^{n}(-t)^{n-1}}{n !}
\end{aligned}
$$

and differentiating term-by-term $\beta_{j}$ times with respect to $t$, we get

$$
\sum_{n=1}^{\infty} \chi_{j}(n) n^{\beta_{j}} e^{-n t}=\sum_{n=\beta_{j}+1}^{\infty} \frac{-B_{\chi_{j}}^{n}(-t)^{n-\beta_{j}-1}}{n \cdot\left(n-\beta_{j}-1\right) !} .
$$

Consequently we have

$$
\lim _{t \rightarrow 0^{+}} F_{\beta}(t)=\prod_{j=1}^{r}\left(-\frac{B_{\chi_{j}}^{\beta_{j}+1}}{\beta_{j}+1}\right)
$$

and hence our assertion follows.

\section{A Consequence.}

Let $P(X) \in \mathbb{R}\left[X_{1}, \ldots, X_{r}\right]$ be a polynomial as given before and $\xi=\left(\xi_{1}, \ldots\right.$, $\left.\xi_{r}\right) \in \mathbb{C}^{r}$ such that $\left|\xi_{j}\right|=1$ and $\xi_{j} \neq 1$ for all $j$. In 1982, P. Cassou-Noguès considered the zeta function

$$
\begin{aligned}
Z(P, \xi, s) & =\sum_{n \in \mathbb{N}^{r}} \xi^{n} P(n)^{-s} \\
& =\sum_{n_{1}=1}^{\infty} \cdots \sum_{n_{r}=1}^{\infty} \xi_{1}^{n_{1}} \cdots \xi_{r}^{n_{r}} P(n)^{-s}, \operatorname{Re} s>\sigma
\end{aligned}
$$

and she proved that

$$
Z(P, \xi,-m)=R\left(P^{m}\right)(\xi)
$$

where

$$
R\left(P^{m}\right)(T)=\sum_{n \in \mathbb{N}^{r}} P^{m}(n) T^{n}
$$


which is a power series and can be realized as a rational function in $T$.

Here we change the dummy variable $n$ and reformulate the above result so that we can use our theorem to give a new proof.

Theorem (P. Cassou-Noguès). Suppose that

$$
Z(P, \xi, s)=\sum_{n_{1}=1}^{\infty} \cdots \sum_{n_{r}=1}^{\infty} \xi_{1}^{n_{1}} \cdots \xi_{r}^{n_{r}} P\left(n_{1}, \ldots, n_{r}\right)^{-s}, \operatorname{Re} s>\sigma .
$$

Then $Z(P, \xi, s)$ has a meromorphic continuation to the whole complex $s$ plane and for any integer $m \geq 0$,

$$
Z(P, \xi,-m)=\lim _{t \rightarrow 0^{+}} \sum_{n \in \mathbb{N}^{r}} \xi^{n} P^{m}(n) e^{-\left(n_{1}+\cdots+n_{r}\right) t} .
$$

Proof. Recall that in the proof of our result, we use only the following two properties of the Dirichlet characters $\chi_{1}, \ldots, \chi_{r}$.

(1) $\chi_{j}$ is a periodic function, $\chi_{j}\left(n+N_{j}\right)=\chi_{j}(n)$ for all $n \in \mathbb{N}$.

(2) $\chi_{j}$ is non-trivial and $\sum_{a=1}^{N_{j}} \chi_{j}(a)=0$.

Thus, in particular, it works for the case $\chi_{j}(n)=e^{2 \pi i n / N_{j}}$ or in general $\chi_{j}(n)=e^{2 \pi i n \eta_{j}}, \eta_{j}$ is a positive rational number such that $0<\eta_{j}<1$.

Now we suppose that $\xi=\left(\xi_{1}, \ldots, \xi_{r}\right)=\left(e^{2 \pi i q_{1}}, \ldots, e^{2 \pi i q_{r}}\right)$ with $0<q_{j}<$ 1. Let $\eta_{k}=\left(\eta_{1}^{(k)}, \ldots, \eta_{r}^{(k)}\right)$ be a sequence of $r$-tuples of rational numbers such that

(1) $0<\eta_{j}^{(k)}<1$ for all $1 \leq j \leq r, k \geq 1$,

(2) $\lim _{k \rightarrow \infty} \eta_{k}=\xi$.

Consider the sequence of zeta functions $\left\{Z_{k}\right\}$ defined by

$$
Z_{k}\left(P, \eta_{k}, s\right)=\sum_{n \in \mathbb{N}^{r}} \eta_{k}^{n} P(n)^{-s}, \text { Re } s>\sigma .
$$

On the half-plane $\operatorname{Re} s>\sigma$, we have

$$
\lim _{k \rightarrow \infty} Z_{k}\left(P, \eta_{k}, s\right)=Z(P, \xi, s) .
$$

Also all the zeta function $Z_{k}\left(P, \eta_{k}, s\right)$ and $Z(P, \xi, s)$ have analytic continuation to the whole complex $s$-plane. So that

$$
\lim _{k \rightarrow \infty} Z_{k}\left(P, \eta_{k},-m\right)=Z(P, \xi,-m) .
$$

By our result

$$
Z_{k}\left(P, \eta_{k},-m\right)=\lim _{t \rightarrow 0^{+}} \sum_{n \in \mathbb{N}^{r}} \eta_{k}^{n} P^{m}(n) e^{-\left(n_{1}+\cdots+n_{r}\right) t},
$$

it follows that

$$
Z(P, \xi,-m)=\lim _{t \rightarrow 0^{+}} \sum_{n \in \mathbb{N}^{r}} \xi^{n} P^{m}(n) e^{-\left(n_{1}+\cdots+n_{r}\right) t} .
$$


The special values $Z(P, \xi,-m)$ can be expressed in terms of special values of the $L$-series

$$
L_{q}(s)=\sum_{n=1}^{\infty} e^{2 \pi i n q} n^{-s}, \quad \operatorname{Re} s>1,0<q<1 .
$$

From

$$
\begin{aligned}
L_{q}(s) \Gamma(s) & =\sum_{n=1}^{\infty} e^{2 \pi i n q} \int_{0}^{\infty} t^{s-1} e^{-n t} d t \\
& =\int_{0}^{\infty} \frac{e^{2 \pi i q} t^{s-1}}{e^{t}-e^{2 \pi i q}} d t, \quad \operatorname{Re} s>1,
\end{aligned}
$$

we conclude that

$$
\begin{aligned}
& L_{q}(-m)=(-1)^{m} m ! \times\left\{\text { the coefficient of } t^{m}\right. \text { in the power series } \\
& \text { expansion at } \left.t=0 \text { of } \frac{e^{2 \pi i q}}{e^{t}-e^{2 \pi i q}}\right\} .
\end{aligned}
$$

In other words,

$$
\sum_{n=1}^{\infty} e^{2 \pi i n q} e^{-n t}=\frac{e^{2 \pi i q}}{e^{t}-e^{2 \pi i q}}=\sum_{m=0}^{\infty} \frac{(-1)^{m} L_{q}(-m) t^{m}}{m !}, \quad|t|<2 \pi q .
$$

Differentiating the above equality $\beta$ times with respect to $t$, we obtain

$$
\sum_{n=1}^{\infty} e^{2 \pi i n q} n^{\beta} e^{-n t}=\sum_{m=\beta}^{\infty} \frac{L_{q}(-m)(-t)^{m-\beta}}{(m-\beta) !} .
$$

Proposition 5. Suppose that

$$
P^{m}(X)=\sum_{|\alpha|=0}^{m p} C_{\alpha} X^{\alpha}
$$

Then

$$
Z(P, \xi,-m)=\sum_{|\alpha|=0}^{m p} C_{\alpha} L_{q_{1}}\left(-\alpha_{1}\right) \cdots L_{q_{r}}\left(-\alpha_{r}\right) .
$$

Proof. Note that

$$
\begin{aligned}
\sum_{n \in \mathbb{N}^{r}} \xi^{n} P^{m}(n) e^{-\left(n_{1}+\cdots+n_{r}\right) t} & =\sum_{|\alpha|=0}^{m p} C_{\alpha} \sum_{n \in \mathbb{N}^{r}} \xi^{n} n^{\alpha} e^{-\left(n_{1}+\cdots+n_{r}\right) t} \\
& =\sum_{|\alpha|=0}^{m p} C_{\alpha} \prod_{j=1}^{r}\left\{\sum_{n=1}^{\infty} e^{2 \pi i n q_{j}} n^{\alpha_{j}} e^{-n t}\right\} .
\end{aligned}
$$


From

$$
\lim _{t \rightarrow 0^{+}} \sum_{n=1}^{\infty} e^{2 \pi i n q_{j}} n^{\alpha_{j}} e^{-n t}=L_{q_{j}}\left(-\alpha_{j}\right),
$$

we get our assertion by the previous theorem.

Now we give expressions for $L_{q}(-m)$. From the power series expansion

$$
\frac{e^{2 \pi i q}}{e^{t}-e^{2 \pi i q}}=\sum_{n=0}^{\infty} \frac{(-t)^{n} \varepsilon_{n}\left(e^{2 \pi i q}\right)}{n !\left(1-e^{2 \pi i q}\right)^{n+1}}, \quad|t|<2 \pi q,
$$

where $\varepsilon_{n}(p)=\sum_{k=1}^{n} A_{n, k} p^{k}$ is the Eulerian polynomials, the coefficients $A_{n, k}$ are the Eulerian numbers which are the numbers of permutations of the chain $\{1<2<\cdots<n\}$ with precisely $k-1$ descents (see, e.g., [4]), we have

$$
L_{q}(-m)=\frac{\varepsilon_{m}\left(e^{2 \pi i q}\right)}{\left(1-e^{2 \pi i q}\right)^{m+1}} .
$$

Meanwhile, we have the following

Proposition 6. Suppose that

$$
P^{m}(X)=\sum_{|\alpha|=0}^{m p} C_{\alpha} X^{\alpha}
$$

then

$$
Z(P, \xi,-m)=\sum_{|\alpha|=0}^{m p} C_{\alpha} \prod_{j=1}^{r} \frac{\varepsilon_{\alpha_{j}}\left(\xi_{j}\right)}{\left(1-\xi_{j}\right)^{\alpha_{j}+1}}
$$

\section{Sums of Products of Generalized Bernoulli Numbers.}

A well-known relation among the Bernoulli numbers is

$$
\sum_{k=1}^{n-1}\left(\begin{array}{l}
2 n \\
2 k
\end{array}\right) B_{2 k} B_{2 n-2 k}=-(2 n+1) B_{2 n}, \quad \text { for } n \geq 2 .
$$

This was found by many authors, including Euler (ref. [5], [8]). Dilcher remarked in [5] that it may be of interest to find formulas of the above type for sums of products of generalized Bernoulli numbers. In the following Proposition 7, we give a closed expression for sums of products of generalized Bernoulli numbers.

Proposition 7. Let $r$ be a positive integer and $\chi_{i}$ be a non-trivial Dirichlet character with conductor $N_{i}$, for $i=1,2, \ldots, r$. Then for any positive 
integer $m$,

$$
\begin{aligned}
& \sum_{\substack{p_{1}+\cdots+p_{r}=m \\
p_{1}, \ldots, p_{r} \geq 0}}^{m}\left(\begin{array}{c}
m \\
p_{1}, \ldots, p_{r}
\end{array}\right) \frac{B_{\chi_{1}}^{p_{1}+1}}{N_{1}^{p_{1}}\left(p_{1}+1\right)} \cdots \frac{B_{\chi_{r}}^{p_{r}+1}}{N_{r}^{p_{r}}\left(p_{r}+1\right)} \\
= & \sum_{a_{1}=1}^{N_{1}} \cdots \sum_{a_{r}=1}^{N_{r}} \chi_{1}\left(a_{1}\right) \cdots \chi_{r}\left(a_{r}\right) \frac{(-1)^{r-1}}{(r-1) !} \\
& \cdot \sum_{j=0}^{r-1}(-1)^{j}\left\{\sum_{k=0}^{j}\left(\begin{array}{c}
r-1-j+k \\
k
\end{array}\right) s(r, r-j+k) \delta^{k}\right\} \frac{B_{m+r-j}(\delta)}{m+r-j},
\end{aligned}
$$

where $\delta=\frac{a_{1}}{N_{1}}+\cdots+\frac{a_{r}}{N_{r}}$ and $s(n, k)$ is the Stirling number of the first kind.

Proof. Consider the zeta function

$$
Z_{r}(s)=\sum_{n_{1}=1}^{\infty} \cdots \sum_{n_{r}=1}^{\infty} \chi_{1}\left(n_{1}\right) \cdots \chi_{r}\left(n_{r}\right)\left(\sum_{j=1}^{r}\left(\prod_{\substack{i=1 \\ i \neq j}}^{r} N_{i}\right) n_{j}\right)^{-s} .
$$

Substitute $n_{i}=a_{i}+N_{i} m_{i}$ where $a_{i}=1, \ldots, N_{i}$ and $m_{i} \geq 0$ for $i=1, \ldots, r$. Thus $Z_{r}(s)$ becomes

$$
\sum_{a_{1}=1}^{N_{1}} \cdots \sum_{a_{r}=1}^{N_{r}} \sum_{m_{1}=0}^{\infty} \cdots \sum_{m_{r}=0}^{\infty}\left(\prod_{i=1}^{r} \chi_{i}\left(a_{i}+m_{i} N_{i}\right) N_{i}^{-s}\right)\left[\sum_{j=1}^{r}\left(m_{j}+\frac{a_{j}}{N_{j}}\right)\right]^{-s} .
$$

Now we let

$$
Z_{B}(s)=\sum_{m_{1}=0}^{\infty} \ldots \sum_{m_{r}=0}^{\infty}\left(\prod_{i=1}^{r} N_{i}\right)^{-s}\left[\sum_{j=1}^{r}\left(m_{j}+\frac{a_{j}}{N_{j}}\right)\right]^{-s} .
$$

Then we can represent the zeta function $Z_{r}(s)$ as

$$
Z_{r}(s)=\sum_{a_{1}=1}^{N_{1}} \cdots \sum_{a_{r}=1}^{N_{r}}\left(\prod_{i=1}^{r} \chi_{i}\left(a_{i}\right)\right) Z_{B}(s) .
$$

From [8] we know that this zeta function $Z_{B}(s)$ has an analytic continuation to the whole complex plane, and the special values at non-positive integers $s=-m$ are given by

$$
Z_{B}(-m)=\left(\prod_{i=1}^{r} N_{i}^{m}\right) \sum_{\substack{p_{1}+\cdots+p_{r}=m+r \\ p_{1}, \ldots, p_{r} \geq 0}} \frac{m !}{p_{1} ! \cdots p_{r} !} \prod_{j=1}^{r} B_{p_{j}}\left(\frac{a_{j}}{N_{j}}\right) .
$$


Using the following identity ([5], Theorem 3)

$$
\begin{gathered}
\sum_{\substack{j_{1}+\cdots+j_{r}=n \\
j_{1}, \ldots, j_{r} \geq 0}}\left(\begin{array}{c}
n \\
j_{1}, \ldots, j_{r}
\end{array}\right) B_{j_{1}}\left(x_{1}\right) \cdots B_{j_{r}}\left(x_{r}\right)= \\
(-1)^{r-1} r\left(\begin{array}{c}
n \\
r
\end{array}\right) \sum_{j=0}^{r-1}(-1)^{j}\left\{\sum_{k=0}^{j}\left(\begin{array}{c}
r-j-1+k \\
k
\end{array}\right) s(r, r-j+k) y^{k}\right\} \frac{B_{n-j}(y)}{n-j},
\end{gathered}
$$

where $y=x_{1}+\cdots+x_{r}$ and $s(n, k)$ are Stirling numbers of the first kind, and we can rewrite $Z_{B}(-m)$ as

$$
\begin{aligned}
\frac{\left(\prod_{i=1}^{r} N_{i}^{m}\right)(-1)^{r-1}}{(r-1) !} & \sum_{j=0}^{r-1}(-1)^{j} \\
& \cdot\left\{\sum_{k=0}^{j}\left(\begin{array}{c}
r-1-j+k \\
k
\end{array}\right) s(r, r-j+k) \delta^{k}\right\} \frac{B_{m+r-j}(\delta)}{m+r-j},
\end{aligned}
$$

where $\delta=\frac{a_{1}}{N_{1}}+\cdots+\frac{a_{r}}{N_{r}}$. Now applying our theorem, the special values at non-positive integers $s=-m$ of the zeta function $Z_{r}(s)$ are

$$
Z_{r}(-m)=\sum_{\substack{p_{1}+\cdots+p_{r}=m \\
p_{1}, \ldots, p_{r} \geq 0}}\left(\begin{array}{c}
m \\
p_{1}, \ldots, p_{r}
\end{array}\right)\left(\prod_{i=1}^{r} \frac{N_{i}^{m-p_{i}} B_{\chi_{i}}^{p_{i}+1}}{p_{i}+1}\right) .
$$

On the other hand, using the equality

$$
Z_{r}(-m)=\sum_{a_{1}=1}^{N_{1}} \cdots \sum_{a_{r}=1}^{N_{r}} \prod_{i=1}^{r} \chi_{i}\left(a_{i}\right) Z_{B}(-m)
$$

and the above values of $Z_{r}(-m)$ and $Z_{B}(-m)$, we get our assertion.

Remark. As special cases we state formulas for sums of products of two, respectively three, generalized Bernoulli numbers.

(1) Let $\chi_{1}, \chi_{2}$ be non-trivial Dirichlet characters with conductors $N_{1}, N_{2}$, respectively. Then for any positive integer $m$,

$$
\begin{aligned}
\sum_{k=0}^{m}\left(\begin{array}{c}
m \\
k
\end{array}\right) \frac{B_{\chi_{1}}^{k+1}}{N_{1}^{k}(k+1)} \frac{B_{\chi_{2}}^{m-k+1}}{N_{2}^{m-k}(m-k+1)} \\
=\sum_{a_{1}=1}^{N_{1}} \sum_{a_{2}=1}^{N_{2}} \chi_{1}\left(a_{1}\right) \chi_{2}\left(a_{2}\right) \\
\quad \cdot\left[\frac{\frac{a_{1}}{N_{1}}+\frac{a_{2}}{N_{2}}-1}{m+1} B_{m+1}\left(\frac{a_{1}}{N_{1}}+\frac{a_{2}}{N_{2}}\right)-\frac{B_{m+2}\left(\frac{a_{1}}{N_{1}}+\frac{a_{2}}{N_{2}}\right)}{m+2}\right] .
\end{aligned}
$$


(2) Let $\chi_{1}, \chi_{2}, \chi_{3}$ be non-trivial Dirichlet characters with conductors $N_{1}$, $N_{2}, N_{3}$, respectively. Then for any positive integer $m$, we have

$$
\begin{aligned}
& \sum_{\substack{p+q+r=m \\
p, q, r \geq 0}}\left(\begin{array}{c}
m \\
p, q, r
\end{array}\right) \frac{B_{\chi_{1}}^{p+1}}{N_{1}^{p}(p+1)} \frac{B_{\chi_{2}}^{q+1}}{N_{2}^{q}(q+1)} \frac{B_{\chi_{3}}^{r+1}}{N_{3}^{r}(r+1)} \\
&=\frac{1}{2} \sum_{a_{1}=1}^{N_{1}} \sum_{a_{2}=1}^{N_{2}} \sum_{a_{3}=1}^{N_{3}} \chi_{1}\left(a_{1}\right) \chi_{2}\left(a_{2}\right) \chi_{3}\left(a_{3}\right) \\
& \cdot\left[\left(\delta^{2}-3 \delta+2\right) \frac{B_{m+1}(\delta)}{m+1}+(3-2 \delta) \frac{B_{m+2}(\delta)}{m+2}+\frac{B_{m+3}(\delta)}{m+3}\right],
\end{aligned}
$$

where $\delta=\frac{a_{1}}{N_{1}}+\frac{a_{2}}{N_{2}}+\frac{a_{3}}{N_{3}}$.

As a final example we consider the Euler numbers $E_{n}, 0 \leq n<\infty$. We have $E_{2 n+1}=0, n \geq 0$, while $E_{2 n}, n \geq 0$, is defined by

$$
\sec x=\sum_{n=0}^{\infty} \frac{(-1)^{n} E_{2 n}}{(2 n) !} x^{2 n}, \quad|x|<\frac{\pi}{2} .
$$

The Euler numbers are special cases of the generalized Bernoulli numbers $B_{\chi}^{n}$ belonging to a residue class character $\chi$. In fact we have

$$
E_{n}=-\frac{2 B_{\eta}^{n+1}}{n+1}, \quad n \geq 0
$$

where $\eta$ is the primitive character with conductor 4 . If we let $r=2$ and the characters $\chi_{1}$ and $\chi_{2}$ in Proposition 7 be the same character $\eta$ (the primitive character with conductor 4 ), then we get an identity which is a special case of Eq. (4.9) in [5].

Proposition 8. For a non-negative integer n, we have the following identity:

$$
\sum_{k=0}^{n}\left(\begin{array}{l}
2 n \\
2 k
\end{array}\right) E_{2 k} E_{2 n-2 k}=\left(2^{2 n+2}-1\right) \frac{2^{2 n+2} B_{2 n+2}}{2 n+2} .
$$

Proof. Let $r=2$ and $\chi_{1}, \chi_{2}$ as indicated above, i.e., $N_{1}=N_{2}=4$. Then

$$
\begin{aligned}
& 4^{-m} \sum_{k=0}^{m}\left(\begin{array}{c}
m \\
k
\end{array}\right) \frac{B_{\chi}^{k+1} B_{\chi}^{m-k+1}}{(k+1)(m-k+1)} \\
& =\sum_{a=1}^{4} \sum_{b=1}^{4} \chi(a b)\left[\frac{\frac{a+b}{4}-1}{m+1} B_{m+1}\left(\frac{a+b}{4}\right)-\frac{B_{m+2}\left(\frac{a+b}{4}\right)}{m+2}\right] \\
& =-\frac{B_{m+1}\left(\frac{1}{2}\right)}{2(m+1)}-\frac{B_{m+2}\left(\frac{1}{2}\right)}{m+2}+\frac{2 B_{m+2}(1)}{m+2}+\frac{B_{m+1}\left(\frac{3}{2}\right)}{2(m+1)}-\frac{B_{m+2}\left(\frac{3}{2}\right)}{m+2} .
\end{aligned}
$$


The left-hand side of the above identity is exactly $4^{-m-1} \sum_{k=0}^{m}\left(\begin{array}{c}m \\ k\end{array}\right) E_{k} E_{m-k}$. Using some basic properties of the Bernoulli polynomials:

$$
\begin{aligned}
B_{n}\left(\frac{3}{2}\right) & =2^{1-n} \cdot n+B_{n}\left(\frac{1}{2}\right), \\
B_{n}\left(\frac{1}{2}\right) & =\left(2^{1-n}-1\right) B_{n}, \\
B_{n}(1-x) & =(-1)^{n} B_{n}(x),
\end{aligned}
$$

the right-hand side of the above identity becomes

$$
2 \cdot\left[1+(-1)^{m+2}-2^{-m-1}\right] \frac{B_{m+2}}{m+2} .
$$

The result follows by setting $m=2 n$.

\section{References}

[1] P. Cassou-Nouguès, Valeurs aux entiers négatifs des fonctions zêta et fonctions zêta p-adiques, Invent. Math., 51 (1979), 25-59.

[2] _ Valeurs aux entiers négatifs des séries de Dirichlet associées a un polynôme, I, J. Number Theory, 14 (1982), 32-64.

[3] _ Séries de Dirichlet et intégrales associées à un polynôme à deux indéterminées, J. Number Theory, 23 (1986), 1-54.

[4] L. Comtet, Advanced Combinatorics, Dordrecht and Boston: Reidel, 1974.

[5] K. Dilcher, Sums of products of Bernoulli numbers, J. Number Theory, 60 (1996), 23-41.

[6] Minking Eie, On a Dirichlet series associated with a polynomial, Proc. Amer. Math. Soc., 110 (1990), 583-590.

[7] _ The special values at negative integers of Dirichlet series associated with polynomials of several variables, Proc. Amer. Math. Soc., 119 (1993), 51-61.

[8] _ A note on Bernoulli numbers and Shintani generalized Bernoulli polynomials, Trans. Amer. Math. Soc., 348 (1996), 1117-1136.

[9] I.M. Gelfand and G.E. Shilov, Generalized Functions, Vol. 1, Academic Press, 1964.

[10] Mathematical Society of Japan, Encyclopedic Dictionary of Mathematics, Cambridge, Mass.: MIT Press, 1993.

[11] Serge Lang, Algebraic Number Theory, Springer-Verlag, 1994.

[12] H. Rademacher, Topics in Analytic Number Theory, in 'Die Grundlehren der Mathematischen Wissenschaften', Band 169, Springer-Verlag, 1973.

[13] T. Shintani, On evaluation of zeta functions of totally real algebraic number fields at non-positive integers, J. Fac. Sci. Univ. Tokyo Sect. IA Math., 23 (1976), 393-417.

[14] L.C. Washington, Introduction to Cyclotomic Fields, Springer-Verlag, 1982. 
[15] D. Zagier, Valeurs des fouctions zêta des corps quadratiques réels aux entiers négatifs, Astérisque, 41-42 (1977), 135-151.

Received July 9, 1999 and revised December 30, 1999. This work was supported by the National Science Foundation of Taiwan, Republic of China.

Department of Accounting and Statistics

Dahan Institute of Technology

Shin-Cheng, Hua-Lian 971

TAIWAN

RePublic of China

E-mail address: kwchen@ms01.dahan.edu.tw

Institute of Applied Mathematics

National Chung Cheng University

Ming-Hsiung, Chia-Yi 621

TAIWAN

Republic OF China

E-mail address: mkeie@math.ccu.edu.tw 